\section{Definition and prevalence of severe and persistent} mental illness

\author{
MIRELLA RUGGERI, MORVEN LEESE, GRAHAM THORNICROFT, \\ GIULIA BISOFFI and MICHELE TANSELLA
}

\author{
Background There is little consistency \\ in how severe mental illness (SMI) is \\ defined in practice, and no operational \\ definitions.
}

\begin{abstract}
Aims Totest two operationalised definitions, based on the National Institute of Mental Health (1987) definition: the first uses three criteria (diagnosis of psychosis; duration of service contact $\geqslant 2$ years; GAF score $\leqslant 50$ ), the second only the last two.
\end{abstract}

\section{Method Annual prevalence rates of SMI in two European catchment areas for each criterion and the criteria combined were calculated.}

Results The first definition produced rates of 2.55 and $1.34 / 1000$ in London and Verona, respectively; the second permitted an additional $0.98 / 1000$ nonpsychotic disorders to be included in Verona.

\section{Conclusions The three-dimensional} definition selects a small group of patients with SMI who have psychotic disorders. The two-dimensional approach allows estimates of SMI prevalence rates which include all forms of mental disorder.

Declaration of interest Funding provided by the University of Verona and the Bethlem and Maudsley NHS Trust.
In recent years a consensus has emerged in many areas, including the UK, that mental health services should be especially targeted at those suffering from severe mental illness (SMI). Several reports on mental health services have indicated deficiencies in this respect (House of Commons Health Select Committee, 1994). In other countries, such as Italy, there is no clear guidance on targeting specific groups among those with mental illness; nevertheless there is an increasing awareness that those with SMI should be given higher priority. However, there is no internationally agreed definition of SMI. Three key issues emerge: how should SMI be defined; what is the prevalence of such morbidity; how can this information best be used for service planning and provision?

A widespread survey in England (Slade $e t$ $a l, 1996)$ found little consistency in how SMI is defined in practice. The most complete review of the subject is that of Schinnar et al (1990), who compared 17 definitions of severe and persistent mental illness used in the USA between 1972 and 1987. They found wide inconsistencies in these definitions: when applied to 222 adult in-patients in Philadelphia, between $4 \%$ and $88 \%$ of patients qualified as having SMI, depending upon the definition selected. The authors concluded that the definition with the widest measure of consensus, and most representative of the middle range of prevalence, was that of the National Institute of Mental Health (NIMH) (1987). This definition categorised individuals as having SMI if they met three criteria: a diagnosis of non-organic psychosis or personality disorder; duration characterised as involving "prolonged illness and long-term treatment" and operationalised as a two-year or longer history of mental illness or treatment; and disability, which was described as including at least three of the eight specified criteria (NIMH, 1987).

The present study is an extension of the work of Schinnar et al (1990), and its aims are to calculate prevalence rates of SMI according to narrow (three-dimensional) and broad (two-dimensional) operationalised definitions of SMI, both derived from the NIMH (1987) definition, and to provide population-based prevalence rates of SMI, defined according to both definitions, in two catchment areas in Europe (South London and South Verona).

\section{METHOD}

The London data are drawn from the baseline assessment of a prospective controlled trial of community mental health services (CMHS) for people with psychotic disorders in an epidemiologically defined area of South London (the PRiSM study) which took place between 1991 and 1992 (Thornicroft et al, 1998). Verona data are drawn from an outcome study conducted in 1994 (the South Verona Outcome Project; Ruggeri et al, 1998a,b) of patients living in an epidemiologically defined area and attending the South Verona CMHS.

\section{Operationalised definitions of SMI}

The narrow definition that we test in this paper (the 'three-dimensional definition') is that a patient has severe mental illness when he or she has the following: a diagnosis of any non-organic psychosis; a duration of treatment of two years or more; dysfunction, as measured by the Global Assessment of Functioning (GAF) scale (American Psychiatric Association, 1987). Specifically, the two levels of dysfunction defined by cut-off points of the GAF are tested: moderate or severe dysfunction (a GAF score of 70 or less, indicating mild symptoms or some difficulty in social, occupation or school functioning); or only severe dysfunction (a GAF score of 50 or less, indicating severe symptoms or severe difficulty in social, occupational or school functioning). The broad definition (the 'two-dimensional definition') is based on the fulfilment of the latter two criteria only.

These definitions differ from the one operationalised by Schinnar et al (1990) in the following respects: psychoses are included, but personality disorders are excluded; the criterion of duration has been simplified, with the exclusion of duration of illness and the inclusion of duration of treatment; a simple operationalisation of the disability criterion was made by using the concept of dysfunction as defined by the GAF. 
The reasons for these changes are the following: the diagnosis of personality disorders has low interrater reliability (Zimmermann, 1994); the duration of treatment can be assessed much more precisely than duration of illness (Schinnar et al, 1990); insufficient information is given on how to operationalise the disability criterion in both the original NIMH (1987) definition and the further work of Schinnar et al. We chose the GAF, despite some limitation in its specificity in measuring disability (Roy-Byrne et al, 1996), because of its simplicity, reliability and widespread use as a proxy measure of disability (Jones et al, 1995).

\section{Characteristics of the catchment areas}

The Nunhead and Norwood sectors (geographical catchment areas) of South London are severely socially deprived areas (see Thornicroft et al, 1998 for more details). The South Verona area of northeast Italy is relatively affluent and predominantly middle class. In 1994 the population aged over 18 was 62 240. The South Verona CMHS is a public service established in 1978 within the newly organised Italian National Health Service, and is the main psychiatric service providing care to South Verona residents (see Tansella et al, 1998 for more details).

\section{Patient inclusion criteria}

\section{Psychotic diagnoses}

This study includes all people with functional psychosis aged over 18 who had an ICD-10 diagnosis of an affective or nonaffective functional psychotic disorder (codes F20-F22, F24, F25, F28-F31, F32.3, F33.3) (World Health Organization, 1992a). Patients with organic psychotic disorders were excluded from the study, as they do not usually use adult mental health services and are cared for by specialist geriatric services.

\section{Non-psychotic diagnoses}

This study includes all South Verona patients aged over 18 with an ICD-10 diagnosis of mental disorder other than functional psychosis.

\section{Assessments}

\section{Diagnosis}

In South London, as part of the PRiSM psychosis study, identification of cases of functional psychotic disorders for the index year (1991-1992) was carried out according to the procedure described in Thornicroft $e t$ al (1998). Briefly, those who had a clinical diagnosis at any time in their lives of any psychotic disorder were identified. Diagnoses were made from case notes by researchers (under the supervision of a psychiatrist) using the Operational Criteria Checklist (OPCRIT, version 3.2; McGuffin et al, 1991) to produce ICD-10 diagnoses. About half were randomly re-assessed using Schedules for Clinical Assessment in Neuropsychiatry (SCAN; World Health Organization, 1992b) interviews conducted by trained psychiatrists. The reliability of this procedure has been demonstrated in another study (McGuffin et al, 1991). In the current study, regular interrater reliability checks were made for both procedures. Of the 566 cases originally identified in the first phase, 514 were considered as 'prevalent' cases in these terms; of these, 511 met the age criterion for the comparison reported here.

In South Verona, diagnosis was established by using the local psychiatric case register (PCR) (Tansella et al, 1998). Diagnoses were made by senior professionals using ICD-10 criteria, and all were reviewed by the director of the PCR. The reliability of this diagnostic procedure is known to be satisfactory (Sytema et al, 1989; Balestrieri et al, 1997).

\section{Service utilisation}

Information was collected from clinical case records in London and the PCR in Verona. Duration of service contact was operationalised as the time elapsed from the date of first contact with any psychiatric service until the start of the respective study period, and dichotomised as either less than two years, or two years or longer.

\section{Global functioning}

In South London, functioning according to the GAF was rated by trained research staff on the basis of information gathered from patients' case notes (using sources in mental health and primary health care services) and from social services records, while in Verona the GAF was completed by the patients' key professionals (psychiatrists or psychologists), who had received appropriate training. In both sites GAF assessments referred to the previous month. All assessors attended a three-hour session which provided a description of the scale and instruction on its use. The good interrater reliability of the GAF when used under routine clinical conditions by members of a community mental health team has been demonstrated in previous studies (Jones et al, 1995). In this study, two interrater reliability exercises were conducted at the end of the training session; interrater reliability was always over 0.90 (intraclass correlation coefficient).

\section{Statistical methods}

Data were analysed using SPSS version 7.0 (SPSS, 1996). Missing values, which were usually a result of sparse information in case notes, were assumed to be missing at random. Annual prevalence rates were then calculated for each combination of criteria for the two- and three-dimensional definitions, on the basis of the proportions of patients with valid data having the required characteristics, assuming independence of categories within each site.

All data for London relate to a one-year period prevalence, whereas Verona used data for dysfunction from patients observed over three months; for this reason, the three-month annual period prevalence rates for Verona were further adjusted by a factor equal to the total number of patients registered in the PCR in the index year divided by the total over three months. We computed $95 \%$ confidence intervals (CIs) for prevalence rates on the basis of the raw unadjusted frequencies, and then applied the appropriate factors.

\section{RESULTS}

\section{Prevalence of psychosis and other mental illness}

Table 1 shows the socio-demographic and service use characteristics of the London and Verona patients. Five hundred and eleven cases of psychosis were identified in a one-year period in London and 212 in Verona, with annual prevalences per thousand inhabitants of $7.84 \quad(95 \%$ CI 7.16-8.52) and 3.41 (95\% CI 2.79-4.02), respectively. Among patients with psychosis in London and Verona, there were $67 \%$ and $74 \%$ with schizophrenia, $6 \%$ and $26 \%$ with affective disorder, and 
Table I Socio-demographic and service utilisation characteristics of patients with psychosis aged over 18 from Nunhead and Norwood (London) and South Verona

\begin{tabular}{|c|c|c|c|}
\hline Characteristic & London $(n=5 I I)^{\prime}$ & South Verona $(n=212)^{\prime}$ & $P^{2}$ \\
\hline \multicolumn{4}{|l|}{ Age (years) } \\
\hline Mean & 42.2 & 47.5 & \\
\hline $95 \% \mathrm{Cl}$ & $40.8-43.6$ & $45.5-49.6$ & $<0.001$ \\
\hline Median & 39 & 46 & \\
\hline \multicolumn{4}{|l|}{ Gender } \\
\hline Male & 251 (49\%) & $98(46 \%)$ & 0.419 \\
\hline Female & $260(51 \%)$ & II4 (54\%) & \\
\hline \multicolumn{4}{|l|}{ Ethnic group } \\
\hline White & 315 (63\%) & $212(100 \%)$ & \\
\hline Non-White & $187(37 \%)$ & 0 & $<0.001$ \\
\hline Missing data & 9 & 0 & \\
\hline \multicolumn{4}{|l|}{ Living situation } \\
\hline Alone & $185(40 \%)$ & 43 (2I\%) & \\
\hline With family & $200(43 \%)$ & $153(73 \%)$ & \\
\hline Sheltered accommodation & 51 (II\%) & $13(6 \%)$ & \\
\hline Other & $25(5 \%)$ & 0 & $<0.001$ \\
\hline Missing data & 50 & 3 & \\
\hline \multicolumn{4}{|l|}{ Employment } \\
\hline Employed & 79 (19\%) & $62(29 \%)$ & \\
\hline Not employed & $340(81 \%)$ & 149 (7I\%) & 0.003 \\
\hline Missing data & 98 & $\mathbf{I}$ & \\
\hline \multicolumn{4}{|l|}{ Duration of contacts } \\
\hline$<2$ years & $58(12 \%)$ & $30(15 \%)$ & \\
\hline$\geqslant 2$ years & $430(88 \%)$ & 173 (85\%) & 0.299 \\
\hline Missing data & 23 & 9 & \\
\hline \multicolumn{4}{|c|}{ In-patient admissions in previous year } \\
\hline None & $329(65 \%)$ & $156(74 \%)$ & \\
\hline At least I & $18 \mid(35 \%)$ & $56(26 \%)$ & 0.018 \\
\hline Missing data & $\mathrm{I}$ & 0 & \\
\hline \multicolumn{4}{|c|}{ Out-patient attendances in previous year } \\
\hline None & $130(27 \%)$ & $46(21.7 \%)$ & \\
\hline At least I & $356(73 \%)$ & $166(78.3 \%)$ & 0.158 \\
\hline Missing data & 25 & 0 & \\
\hline \multicolumn{4}{|l|}{ Compulsory admission } \\
\hline None & 157 (37\%) & 163 (77\%) & $<0.001$ \\
\hline At least I & $265(63 \%)$ & 49 (23\%) & \\
\hline Missing data & 89 & 0 & \\
\hline
\end{tabular}

I. Number of patients identified in one year.

2. Chi-squared, Fisher's exact test or $t$-test.

$27 \%$ and $1 \%$ with an ICD-10 diagnosis of other non-organic psychotic syndrome (F28) or unspecified non-organic psychosis (F29), respectively. The higher number of unspecified diagnoses in London was due to a greater proportion of case notes with incomplete clinical information. This problem did not exist in Verona because diagnoses were made for all patients as part of routine clinical practice.

In South Verona, 711 patients with a diagnosis of mental disorder other than psychosis were identified by the PCR in the index year, with an annual period prevalence per thousand inhabitants of 11.42 (95\% CI 10.58-12.26). Fifteen per cent of these patients had an ICD-10 diagnosis of personality disorder, $20 \%$ of neurotic somatoform disorder, and 39\% of neurotic depression, while $17 \%$ had other diagnoses (including F10 alcoholism and F11-19, F55 drug misuse), and $8.5 \%$ had missing data.

\section{Assessment of duration and dysfunction}

All identified cases of psychosis in the index year in London underwent the social functioning assessment. Assessments of duration and dysfunction were missing in 23 and 108 cases, respectively, usually because of very brief case records. Possible bias due to the large proportion of missing values was investigated by comparing those with and without case-note GAF scores using the GAF from an interview approximately one year later (available from the main PRiSM study) for a randomly chosen subsample. Those with and without missing case-note GAF scores had very similar mean interview GAF scores (59.2 compared with $63.9, P=0.12$ ).

In Verona only the patients of the catchment area attending the South Verona CMHS $(n=542)$ underwent the social functioning assessment. In this group most missing data resulted from the study design, which is based on routine clinical assessments made by the key professionals and not by researchers. Global Assessment of Functioning assessments were missing in 187 patients: in 49 cases GAF assessments were missing because the patients, although in contact with other professionals such as nurses or social workers, had not been seen by a doctor or psychologist in the threemonth index period; in the remaining 138 cases assessments were missing because, owing to lack of time, the key professionals could not complete the GAF assessment after their visit. Patients with missing assessments had had significantly fewer contacts with the service in the previous year $(P<0.01)$, but did not differ from the patients assessed for any other sociodemographic or service utilisation characteristic.

\section{Criteria based on duration and dysfunction}

Table 2 shows the application of the operationalised criteria based on duration and dysfunction, both individually and combined, to all patients with psychotic 
Table 2 Annual period prevalence rates of severe and persistent mental illness in Nunhead and Norwood (London) and South Verona using operationalised criteria applied to patients with and without psychotic disorders

\begin{tabular}{|c|c|c|c|c|c|c|c|c|c|}
\hline \multirow[b]{3}{*}{$\begin{array}{l}\text { Operationalised } \\
\text { criteria }\end{array}$} & \multirow{2}{*}{\multicolumn{3}{|c|}{$\begin{array}{l}\text { London (65 I50 inhabitants over } 18) \\
\text { With psychosis }(n=5 I I)^{\prime}\end{array}$}} & \multicolumn{6}{|c|}{ South Verona (62 240 inhabitants over 18) } \\
\hline & & & & \multicolumn{3}{|c|}{ With psychosis $(n=212)^{2}$} & \multicolumn{3}{|c|}{ Without psychosis $(n=7 I I)^{3}$} \\
\hline & $\begin{array}{l}\text { Rate per } \\
1000\end{array}$ & $95 \% \mathrm{Cl}$ & $\begin{array}{l}\text { Estimated percentage } \\
\text { of patients }\end{array}$ & $\begin{array}{l}\text { Rate per } \\
1000\end{array}$ & $95 \% \mathrm{Cl}$ & $\begin{array}{l}\text { Estimated percentage } \\
\text { of patients }\end{array}$ & $\begin{array}{l}\text { Rate per } \\
1000\end{array}$ & $95 \% \mathrm{Cl}$ & $\begin{array}{c}\text { Estimated percentage } \\
\text { of patients }\end{array}$ \\
\hline \multicolumn{10}{|l|}{ Dysfunction } \\
\hline $\mathrm{GAF} \leqslant 70$ & 6.31 & $5.62-6.99$ & $80 \%$ & 2.85 & $2.29-3.42$ & $84 \%$ & 7.70 & $6.50-0.89$ & $67 \%$ \\
\hline $\mathrm{GAF} \leqslant 50$ & 2.76 & $2.31-3.22$ & $35 \%$ & $\mathrm{I} .5 \mathrm{I}$ & $1.10-1.93$ & $44 \%$ & 1.60 & $1.05-2.14$ & $14 \%$ \\
\hline Duration $\geqslant 2$ years & 6.91 & $6.26-7.56$ & $88 \%$ & 3.01 & $2.53-3.49$ & $88 \%$ & 6.99 & $6.07-7.92$ & $62 \%$ \\
\hline \multicolumn{10}{|l|}{ Dysfunction/duration } \\
\hline $\mathrm{GAF} \leqslant 70, \geqslant 2$ years & 5.67 & $4.95-6.35$ & $72 \%$ & 2.45 & $1.92-2.98$ & $72 \%$ & 4.32 & $3.48-5.16$ & $38 \%$ \\
\hline $\mathrm{GAF} \leqslant 50, \geqslant 2$ years & 2.55 & $2.11-3.00$ & $32 \%$ & 1.34 & $0.95-1.73$ & $40 \%$ & 0.98 & $0.58-1.39$ & $9 \%$ \\
\hline
\end{tabular}

GAF, score on the Global Assessment of Functioning scale.

I. PRiSM study, case identification over one year. Basis for the estimation of annual rates: 403 valid cases for dysfunction, 488 for duration and 390 for both diagnosis and duration. 2. Registered (psychiatric case register) in one year; 177 registered in three months. Basis for estimation of three-month rates: II7 valid cases for dysfunction, I7I for duration, II 3 for both duration and dysfunction. Rates adjusted to one year.

3. Registered (psychiatric case register) in one year; 401 registered in three months. Basis for estimation of three-month rates: 236 valid cases for dysfunction, $36 \mathrm{I}$ for duration, 228 for both duration and dysfunction. Rates adjusted to one year.

and non-psychotic disorders identified in the index period in London and Verona. Despite the overall differences in prevalence, the data for psychosis show that the relative proportions of patients with SMI for each criterion of the operationalised definition were very similar between the two sites, although there is some weak evidence for a higher proportion of patients with severe dysfunction in Verona (44\% $v$. $35 \%, P=0.08)$. Log-linear analysis confirmed this finding, but showed no evidence of association between duration and dysfunction at either site.

Among the patients with non-psychotic disorders, those with an ICD-10 diagnosis of personality disorder or alcohol and drug misuse had a significantly higher percentage of SMI than patients with neurotic disorders; specifically, a longer duration of treatment (over $70 \%$ with duration of over two years in patients with personality disorder and other diagnoses $v .60 \%$ in patients with a neurotic disorder) and more severe dysfunction (around $35 \% \quad v$. less than $10 \%$ ) were found. Owing to the small number of cases in each category, the possible combination of criteria was not assessed for these separate diagnostic categories.

\section{Application of combined criteria}

Table 3 shows the application of both twoand three-dimensional definitions in all patients with a diagnosis of mental disorder
Table 3 Annual period prevalence rates of severe and persistent mental illness in South Verona using operationalised criteria applied to all patients

\begin{tabular}{|c|c|c|c|c|c|}
\hline \multirow{2}{*}{\multicolumn{2}{|c|}{ Operationalised criteria }} & & \multicolumn{3}{|c|}{$\begin{array}{l}\text { South Verona (62 } 240 \text { inhabitants over 18) } \\
\qquad(n=933)^{\prime}\end{array}$} \\
\hline & & & $\begin{array}{l}\text { Rate per } \\
1000\end{array}$ & $95 \% \mathrm{Cl}$ & $\begin{array}{c}\text { Estimated } \\
\text { percentage of all cases }\end{array}$ \\
\hline \multicolumn{6}{|c|}{ One-dimensional definitions } \\
\hline \multirow[t]{6}{*}{$\begin{array}{r}\text { Diagnosis of } \\
\text { psychosis }\end{array}$} & & & 3.41 & $2.79-4.02$ & $23 \%$ \\
\hline & Dysfunction & & & & \\
\hline & $\mathrm{GAF} \leqslant 70$ & & 10.54 & $9.22-11.86$ & $70 \%$ \\
\hline & $\mathrm{GAF} \leqslant 50$ & & 3.11 & $2.43-3.79$ & $21 \%$ \\
\hline & & Duration & & & \\
\hline & & $\geqslant 2$ years & 10.00 & $8.96-11.04$ & $67 \%$ \\
\hline \multicolumn{6}{|c|}{ Two-dimensional definitions } \\
\hline \multicolumn{6}{|c|}{ Dysfunction } \\
\hline Diagnosis of & $\mathrm{GAF} \leqslant 70$ & & 2.85 & $2.29-3.42$ & $19 \%$ \\
\hline psychosis & $\mathrm{GAF} \leqslant 50$ & & I.5I & $1.10-1.93$ & $10 \%$ \\
\hline Diagnosis of & & Duration & & & \\
\hline psychosis & & $\geqslant 2$ years & 3.01 & $2.53-3.49$ & $20 \%$ \\
\hline & Dysfunction & Duration & & & \\
\hline & GAF $\leqslant 70$ & $\geqslant 2$ years & 6.77 & $5.78-7.77$ & $45 \%$ \\
\hline & $\mathrm{GAF} \leqslant 50$ & $\geqslant 2$ years & 2.33 & I.77-2.89 & $16 \%$ \\
\hline \multicolumn{6}{|c|}{ Three-dimensional definition } \\
\hline & Dysfunction & Duration & & & \\
\hline Diagnosis of & $\mathrm{GAF} \leqslant 70$ & $\geqslant 2$ years & 2.45 & $1.92-2.98$ & $16 \%$ \\
\hline psychosis & $\mathrm{GAF} \leqslant 50$ & $\geqslant 2$ years & 1.34 & $0.95-1.73$ & $9 \%$ \\
\hline
\end{tabular}

GAF, score on the Global Assessment of Functioning Scale.

I. 933 patients registered in one year; 578 in three months. Basis for estimation of three-month rates: 353 valid cases for dysfunction, 532 for duration, 34 I for both duration and dysfunction. Rates adjusted to one year. 
identified in the index period in the South Verona area. Prevalence rates of SMI vary widely depending on the criteria applied, with low discrimination by the separate criteria of moderate dysfunction and long duration (taken individually or combined) and higher discrimination by the criteria of diagnosis and severe dysfunction. Application of the two-dimensional definition criteria based on severe dysfunction and duration gives a total prevalence rate of SMI of 2.33 per thousand for all disorders; it is noteworthy that of these only $58 \%$ are cases of psychosis.

\section{DISCUSSION}

This is the first epidemiologically based study in Europe to operationalise and apply the NIMH (1987) criteria for SMI. The advantages of the current study over previous work are that the definition used is fully operationalised for each criterion, and so the study can be easily replicated and prevalence rates in other settings calculated, and that the type of operationalisation proposed is based on easily obtainable data, and so can be of widespread use.

\section{Prevalence of SMI among patients with psychosis}

The annual period prevalence of psychosis in South Verona (3.41 per thousand inhabitants) is similar to that found in previous studies in the rest of Italy (Balestrieri et al, 1992) and other European countries and the USA (Robins \& Regier, 1991). The higher rate in South London (7.84 per thousand inhabitants) found in this study is in agreement with other findings for inner London (Johnson et al, 1997) and is consistent with a recent psychiatric morbidity survey which found annual period prevalence rates of between 2.0 and 9.0 per thousand inhabitants in the population throughout Britain (Meltzer et al, 1996). These higher rates are partly due to the higher proportion of ethnic minorities, who more frequently suffer from schizophrenia (McCreadie, 1982).

When the three-dimensional definition (functional psychosis, duration of treatment longer than two years, and a severe dysfunction lasting for at least one month) is applied, prevalence rates of SMI among patients with psychosis are higher in south London than in south Verona (2.55 v. 1.34 per thousand inhabitants). The difference in prevalence of SMI in the two areas matches in general terms the difference in prevalence of psychosis noted above. However, the relative proportion of SMI among those with psychosis is somewhat lower in London than in Verona (31\% v. 40\%).

The results show that in patients with psychosis, the more restrictive criterion for severity of illness is the high severity of dysfunction (a GAF score of 50 or less), since the majority of patients with psychosis had a long duration (88\% in both areas) and at least a moderate (a GAF score of 70 or less) level of dysfunction $(80 \%$ and $84 \%$ in London and Verona, respectively).

\section{Prevalence of SMI among patients with non-psychotic disorders}

In South Verona the total prevalence of non-psychotic disorders treated by psychiatric services was 11.42 per thousand inhabitants. When the full set of twodimensional definitions has to be fulfilled, the prevalence of SMI among patients with non-psychotic disorders is 0.98 per thousand inhabitants $(9 \%)$. The criteria of duration and dysfunction both play an important role in the selection of patients.

\section{Prevalence of SMI among patients with any mental disorder attending adult services}

The total population-based annual prevalence of SMI patients in South Verona is 1.34 per thousand when we apply all criteria of the three-dimensional definition and 2.33 per thousand when all criteria of the two-dimensional definition are applied. The former definition is therefore more powerful in selecting a smaller subgroup of patients with SMI from among all patients with any mental disorder.

According to the two-dimensional definition, in Verona $40 \%$ of all patients with psychosis and $9 \%$ those without have SMI. When considering all patients with a diagnosis of mental disorder, the SMI group according to the two-dimensional definition is composed of $58 \%$ with psychosis and $42 \%$ with non-psychotic disorders. Is it legitimate not to consider such a large proportion of patients (people with non-psychotic disorders) when planning services dedicated to SMI?

\section{Limitations of the study}

This study has a number of limitations. The annual total prevalence rates were accurately estimated, albeit with a possible slight underestimation in London, but there were some missing values for the definitions based on dysfunction. Although there was no evidence that this caused major problems, it would be preferable to minimise bias when using the operationalised criteria to assess prevalence, and this study suggests some improvements which could be made.

For interview data, one improvement would be to make a GAF assessment for a known proportion of all types of patients, including those who would not normally be seen frequently. Weighted methods of analysis can then be used to combine their results with those of routine attenders (see, for example, Dunn et al, 1999). With regard to case-note data, the London results suggest that case-note assessments are acceptable where patients are not seen in person, and that missing data do not lead to major bias. However, further research is needed to eliminate specific bias in GAF assessments based on case notes rather than interviews. Another limitation is that the reliability of GAF assessment, as well as that of the ICD-10 diagnostic coding, is based on previous work done by our group and other groups, but has not been assessed within this study.

A further possibility of bias (overestimation) in SMI, resulting from dysfunction being measured cross-sectionally, might have occurred. This was also the case for duration, since cross-sectional samples are more likely to sample long-duration than short-duration illnesses. However, the much higher degree of feasibility of a crosssectional assessment compared with a longitudinal one suggests that this procedure is to be preferred despite its limitations.

This study did not consider the whole range of mental illnesses in both areas, since in London only cases of functional psychosis were included in the study. Moreover, the fact that in London some of the diagnoses were made from clinical records may have caused an increase in the number of unspecified cases of psychosis; it has been estimated that there was a $3 \%$ underestimation of prevalence as a result of cases having neither adequate case notes nor later interview.

In the group of patients with psychosis a refined diagnostic breakdown was not 
possible, hence the relative contribution of schizophrenia and affective psychosis in this group has not been explored. Diagnostic breakdown was also not possible in the group of patients with non-psychotic disorders in Verona, owing to the smaller number of cases in several categories. An exploratory analysis showed that patients with a diagnosis of personality disorder, alcoholism or drug misuse may suffer from SMI more frequently than the other diagnostic groups. This deserves further investigation.

Another limitation of this study is that two different methods of case identification were employed at the two sites - a population-based method in London and a case register method in Verona. Data in South London reflect the general population prevalence, while data in South Verona are representative of the treated prevalence in specialist services only (all hospitals, both public and private, as well as community psychiatric services). It is well known that treated cases are a selection of community cases, but we also know that severity of illness is the main selective factor in passing from having a mental disorder to searching for specialist treatment for that disorder. According to Olfson \& Klerman (1992), "mental health care utilisation is a reasonable proxy for psychiatric distress and is therefore an acceptable criterion for validating the presence of psychiatric disorder". Moreover, in Italy the vast majority of patients with a diagnosis of psychosis are treated in public psychiatric services or in private hospitals (Balestrieri et al, 1994). All these institutions report to the South Verona PCR, so PCR estimates of prevalence of psychosis may be considered reasonably accurate; hence the comparison with London prevalence rates is appropriate. For people with nonpsychotic disorders, PCR prevalence rates are certainly an underestimation of the true prevalence; even so, we consider that the vast majority of the more severely ill people with non-psychotic disorders are included in the estimates.

Finally, some significant differences between the English and Italian samples were found for age, ethnicity, living situation and service utilisation. These differences derive both from the different sociocultural contexts and from the different models of psychiatric care applied in the two settings; however, they did not relate to the percentages of SMI in the two areas.

\section{CLINICAL IMPLICATIONS}

More than one-third of the patients with a diagnosis of functional psychosis have severe mental illness (SMI).

- Among the patients who are defined as having SMI, about $40 \%$ are affected by a non-psychotic mental disorder.

The definition tested is fully operationalised and based on easily obtainable data, so the study can be replicated and prevalence rates in other settings can be calculated.

\section{LIMITATIONS}

- The study did not consider the whole range of mental illnesses in both London and Verona, the two areas studied, since in London functional psychosis cases only were included.

- Two methods of case identification were employed: a population-based method in London and a case-register method in Verona.

- Further testing of the validity and reliability of the definition of SMI and its diagnostic criteria is needed.

MIRELLA RUGGERI, PhD, Dipartimento di Medicina e Sanità Pubblica, Sezione di Psichiatria, Università di Verona, Ospedale Policlinico, Verona, Italy; MORVEN LEESE, PhD, GRAHAM THORNICROFT, PhD, Section of Community Psychiatry (PRiSM), Institute of Psychiatry, London; GIULIA BISOFFI, Dr Stat, MICHELE TANSELLA, MD, Dipartimento di Medicina e Sanità Pubblica, Sezione di Psichiatria, Università di Verona, Ospedale Policlinico, Verona, Italy

Correspondence: Dr Mirella Ruggeri, Dipartimento di Medicina e Sanita' Pubblica, Sezione di Psichiatria, Università di Verona, 37I34 Verona, Italy. Tel: +39-045-807444I ; fax: +39-045-58587I ; e-mail: mruggeri@borgoroma.univr.it

(First received 14 June 1999, final revision 23 December 1999, accepted 23 December 1999)

\section{Implications of the study}

This study has several implications. On a practical level it has shown the feasibility of applying simple definitions of SMI in two different situations, neither of which was originally designed for prevalence estimation, namely a population-based research project and a case-register project. Annual period prevalence rates of SMI using the three-dimensional model have been compared in two areas in Europe which differ both in the social context and in the type of psychiatric care provided. Despite the limitations of the study mentioned above, these data could provide the first step toward extrapolation to national estimates of SMI throughout Great Britain and Italy and comparison with prevalence of SMI in other countries. In spite of the difference in the annual prevalence rates of SMI, the proportions of SMI among cases with psychosis are similar. This finding, if confirmed in other areas, suggests that there is a type of cross-cultural stability in the proportion of functional psychosis patients who have SMI, which is independent of local prevalence rates of mental illness and could be used for planning.

Epidemiological data collected in one of the sites (Verona) suggest that the use of the two-dimensional definition, with a combination of the criteria of duration of treatment and severe dysfunction, is preferable to the three-dimensional version. This is because the two-dimensional definition does not discriminate against patients with severe non-functional psychotic disorders, and because it allows further research to explore the burden placed on services and carers by patients with severe and enduring non-psychotic mental illnesses. 
Future research priorities include testing the validity of these definitions and checking whether those patients who are identified as having SMI at a baseline point are those who suffer more and will cause more burden subsequently, and whether there are differences in the burden and disability profiles between SMI patients with and without psychosis.

\section{REFERENCES}

American Psychiatric Association (1987) Diagnostic and Statistical Manual of Mental Disorders (3rd edn, revised) (DSM-III-R). Washington, DC: APA

Balestrieri, M., Micciolo, R., De Salvia, D., et al (1992) Confronti e prospettive nella utilizzazione dei Registri Psichiatrici dei Casi. Epidemiologia e Psichiatria Sociale, I, 133-148.

_ , Bon, M. G., Rodriguez-Sacristan, A., et al (1994) Pathways to psychiatric care in South Verona, Italy. Psychological Medicine, 24, 64I-649.

_ , Rucci, P. \& Nicolau, S. (1997) Gender-specific decline and seasonality of births in operationally defined schizophrenics in Italy. Schizophrenia Research, 27, 73-81.

Dunn, G., Pickles, A., Tansella, M., et al (1999) Two phase epidemiological surveys in psychiatric research. British Journal of Psychiatry, 174, 95-100.

House of Commons Health Select Committee (1994) First Report of the Health Committee: Better Off in the Community? The Care of People who are Seriously Mentally III. London: HMSO.
Johnson, S., Brooks, L. \& Thornicroft, G. (1997) London's Mental Health. London: King's Fund.

Jones, S. H., Thornicroft, G., Coffey, M., et al (1995)

A brief mental health outcome scale. Reliability and validity of the Global Assessment of Functioning (GAF). British Journal of Psychiatry, 166, 654-659.

McCreadie, R. (1982) The Nithsdale schizophrenia survey: I. Psychiatric and social handicaps. British Journal of Psychiatry, 140, 582-586.

McGuffin, P., Farmer, A. \& Harvey, I. (1991) A polydiagnostic application of operational criteria in studies of psychotic illness. Development and reliability of the OPCRIT system. Archives of General Psychiatry, 48, 764-770.

Meltzer, H., Gill, B., Petticrew, M., et al (1996) OPCS Surveys of Psychiatric Morbidity in Great Britain. London: HMSO

National Institute of Mental Health (1987) Towards a Model for a Comprehensive Community-Based Mental Health System. Washington, DC: NIMH.

Olfson, M. \& Klerman, G. L. (1992) Depressive symptoms and mental health service utilization in a community sample. Social Psychiatry and Psychiatric Epidemiology, 27, 161-167.

Robins, L. N. \& Regier, D. A. (eds) (1991) Psychiatric Disorders in America: the Epidemiologic Catchment Area Study. New York: Free Press.

Roy-Byrne, P., Dagadakis, C., Unutzer, J., et al (1996) Evidence for limited validity of the revised Global Assessment of Functioning Scale. Psychiatric Services, 47 864-866

Ruggeri, M., Biggeri, A., Rucci, P., et al (1998a) Multivariate analysis of outcome of mental health care using graphical chain models. The South Verona Outcome Project I. Psychological Medicine, 28, |421-1431.
_, Riani, M., Rucci, P., et al (1998b) Multidimensional assessment of outcome in psychiatry: the use of graphical displays. The South Verona Outcome Project 2. International Journal of Methods in Psychiatric Research, 7, $186-198$.

Schinnar, A. P., Rothbard, A. B., Kanter, R., et al (1990) An empirical literature review of definitions of severe and persistent mental illness. American Journal of Psychiatry, 147, 1602-1608.

Slade, M., Powell, R. \& Strathdee, G. (1996) Current approaches to identifying the severely mentally ill. Social Psychiatry and Psychiatric Epidemiology, 32. 177-184.

SPSS (1996) SPSS for Windows: Base System User's Guide. Release 7.0. Chicago, IL: SPSS Inc.

Systema, S., Giel, R., ten Horn, G. H., et al (1989) The reliability of diagnostic coding in psychiatric case registers. Psychological Medicine, 9, 999-1006.

Tansella, M., Amaddeo, F., Burti, L., et al (1998) Community-based mental health care in Verona, Italy. In Mental Health in our Future Cities (eds D. Goldberg \& G. Thornicroft), pp. 239-262. London: Erlbaum (UK), Taylor \& Francis (Psychology Press).

Thornicroft, G., Strathdee, G., Phelan, M., et a (1998) Rationale and design. PRiSM Psychosis Study I. British Journal of Psychiatry, 173, 363-370.

World Health Organization (1992a) The Tenth Revision of the International Classification of Diseases and Related Health Problems (ICD-10). Geneva: WHO.

- (1992b) Schedules for Clinical Assessment in Neuropsychiatry. Geneva: WHO.

Zimmermann, M. (1994) Diagnosing personality disorders: a review of issues and research methods. Archives of General Psychiatry, 5I, 225-245. 